\title{
La modelación matemática en la ingeniería de dieseño ${ }^{1}$
}

Mathematical modeling in engineering of desing

Modelagem matemática em engenharia de desing

Recibido: mayo de 2013

Aceptado: agosto de 2013
Paula Andrea Rendón Mesa ${ }^{2}$

Pedro Vicente Esteban Duarte ${ }^{3}$

Jhony Alexander Villa-Ochoa ${ }^{4}$

\section{Resumen}

Se pretende, en esta comunicación, informar algunos aspectos que se vienen adelantando en una investigación correspondiente a la formación del Doctorado en Educación de la Universidad de Antioquia. Se pretende discutir cómo la Modelación Matemática se puede convertir en una herramienta de formación para los ingenieros, propiamente en Ingeniería de Diseño. Se describe la forma cómo se realiza el trabajo en el transcurso del semestre y se ejemplifica dando a conocer avances de los resultados obtenidos. Finalmente, se plantea la necesidad de pensar la Modelación Matemática, como una alternativa donde el estudiante construya una "realidad" y responda a las exigencias actuales de tener un saber específico aplicado a un contexto, generando una articulación entre el saber matemático y el saber propio de la Ingeniería de Diseño.

Palabras clave: Modelación Matemática; Ingeniería de Diseño; Contexto.

\begin{abstract}
The aim, in this paper, report some aspects that is underway in an investigation related to the formation of the Doctorate in Education from the University of Antioquia. The aim is to discuss how mathematical modeling can be converted into a training tool for engineers, properly in Design Engineering. It describes how the work is performed in the course of the semester and is exemplified by publicizing progress results. Finally, there is the need to think the Mathematical Modeling as an alternative where the student builds a "reality" and meets the current requirements of having a specific knowledge applied to a context, creating a link between mathematical knowledge and knowledge itself Design Engineering.
\end{abstract}

Keywords: Cognitive Processes, Mathematical Modeling, Design Engineering, Context, Analysis and Reflection on teaching.

1 Artículo de Investigación

2 Universidad de Antioquia. Contacto: rendonmesa@hotmail.com;

3 Universidad EAFIT. Contacto: pesteban@eafit.edu.co

4 Universidad de Antioquia. Contacto: javo@une.net.co 


\section{Resumo}

O objetivo, neste artigo, relatar alguns aspectos que está em andamento em uma investigação relacionada com a formação do Doutorado em Educação da Universidade de Antioquia. O objetivo é discutir como a modelagem matemática pode ser convertido em uma ferramenta de treinamento para engenheiros, corretamente em engenharia de projeto. Ele descreve como o trabalho é realizado no decorrer do semestre e é exemplificado pela publicação de resultados de progresso. Finalmente, há a necessidade de pensar a Modelagem Matemática como uma alternativa onde o aluno constrói uma "realidade" e atende aos requisitos atuais de ter um conhecimento específico aplicado a um contexto, a criação de uma ligação entre o conhecimento eo próprio conhecimento matemático projeto de Engenharia.

Palavras chave: processos cognitivos, Modelagem Matemática, Engenharia de Concepção, Contexto, Análise e Reflexão sobre o ensino.

\section{Las matemáticas en ingeniería}

Es bastante común que al interior del aula de matemáticas, se consideren las aplicaciones de esta ciencia en cualquier contexto. Sin embargo, qué el docente y el estudiante reconozcan cuándo y dónde hay matemáticas, es algo que implica una comprensión de la "situación real" que se está analizando. En la formación de un profesional, en particular de un ingeniero, esta situación no es lejana, por el contrario, actualmente se requiere de la integración del saber con el contexto, puesto que se espera que para su desempeño profesional y laboral tengan herramientas para resolver problemas propios de su área de formación. Estas ideas plantean diversos retos a la formación matemática de los futuros profesionales, como son la búsqueda de conexiones entre los contextos propios del estudiante, afines a su campo de formación y las matemáticas mismas. Es por esto que, en el marco del Doctorado en Educación de la Universidad de Antioquia, se pretende adelantar una investigación en la que se integre la Modelación Matemática como una herramienta de formación durante los estudios universitarios.

Para Biembengut \& Hein (2004) con la modelación "el aprendizaje se hace más rico, considerando que el alumno no sólo aprende matemática inserta en el contexto de otra área de conocimiento, sino que también despierta su sentido crítico y creativo" (p. 107). Esto hace ver el contexto como herramienta fundamental para la apropiación de conocimientos matemáticos como lo establecen Masingila et al. (1996), Greer (1997) y Kim (2011) entre otros.

Estas circunstancias permiten dar respuesta a las exigencias actuales en la formación de ingenieros, que plantea la necesidad de que las matemáticas en el aula de clase, dejen de ser solamente un aspecto teórico y algorítmico y es por esto que la Modelación Matemática puede ser el recurso que reconoce el contexto como elemento aportante que dota de significado el proceso de aprendizaje. Esto conlleva a la vinculación de un sentido de realidad que permite, tanto al estudiante como al docente re-significar sus planteamientos, reconociendo una mejor integración entre los elementos teóricos y prácticos (J. A. Villa-Ochoa \& Jaramillo, 2011).

\section{El camino seguido en ingeniería de diseño}

En la Universidad EAFIT, en el programa de Ingeniería de Diseño se imparte un curso llamado Modelación Matemática. Este espacio de formación tiene como propósito acerca a los estudiantes a reconocer las conceptualizaciones matemáticas que existen en cualquier objeto creado. Bajo este propósito, el trabajo se desarrolla en tres momentos a lo largo del semestre. El primero, llamado fase de exploración, conduce a indagar sobre los conceptos que surgen en diferentes escenarios de la "vida 
real". Un segundo momento, la fase de investigación guiada, permite definir un referente de diseño y plantear una propuesta de modelo vinculando diferentes aspectos estéticos y conceptuales de la matemática y el diseño; y la tercera fase, denominada proyecto final de síntesis, donde se construye y valida el modelo pensado en la fase anterior.

El estudio de los resultados de la aplicación de estas fases al interior del aula, han posibilitado reconocer como las conceptualizaciones de los estudiantes frente a las matemáticas y a la Ingeniería de Diseño se encuentran desarticuladas. Por lo tanto, es necesario encontrar actividades que permita articular estas ramas del conocimiento. A continuación expondremos un episodio para ejemplificar lo ocurrido y generar así algunas reflexiones.

\section{Un episodio}

En este apartado, se presentan los productos desarrollados por un estudiante durante los tres momentos de intervención, dando cuenta de las conceptualizaciones alcanzadas durante el proceso.

Fase de exploración. Los estudiantes en esta fase del trabajo de aula, seleccionan diversas imágenes y en ellas reconocen conceptos matemáticos implícitos. Uno de ellos selecciono un carro tanque y lo describió de la siguiente forma: El Camión: Aquí se hacen presentes los cilindros, en el tanque, obviamente. Además, en las llantas que son cilindros de altura más pequeña y en los tubos de los mofles. También, se ven dos cuadrados en las luces y varios rectángulos. Estas conceptualizaciones dan cuenta del reconocimiento de formas, asociándolas con figuras geométrica conocidas, pero no se relacionan con el funcionamiento del objeto ni lo que ello implica en su estructura global de diseño. La vinculación de la matemática en esta fase, queda ligada a lo estético, dejando de lado como esta ciencia está relacionada con las condiciones de funcionalidad del mismo.

Fase de investigación. Para esta fase, el estudiante seleccionó como referente de creación el camión y sobre esta idea consolida una nueva que le permita generar un diseño. En este caso él formuló la siguiente iniciativa de diseño: Generar en los camiones una bisagra superior y una inferior ubicadas en el centro del tráiler (Fig. 1), acompañadas de un eje de 2 ruedas, con un ángulo de giro restringido mediante sistemas hidráulicos dirigidos desde la cabina del conductor. El camión es un medio de transporte, vehículo muy pesado principalmente por sus piezas tan voluminosas por esta razón los camiones no hacen giros fácilmente. En el diseño se pretende dar una solución a esto, por medio de un sistema en su tráiler, que le dará más flexibilidad al camión y, además, de esto puede transportar mayor cantidad de carga. Este doble tráiler le posibilita a los conductores un mejor desempeño en las carreteras de nuestro país.

Fig. 1. Dibujo de iniciativa de diseño en la fase de investigación.

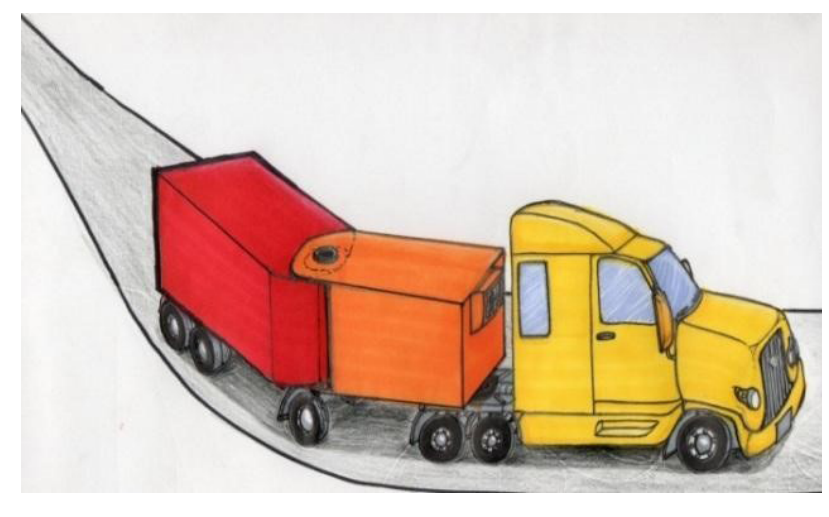

Fuente: Elaboración propia

Esta fase se percibe como la creatividad y el ingenio no se aproximan a la matemática, donde ella puede convertirse en aliado estratégico para apuntalar la idea y con la definición de variables apropiadas y la vinculación de otras áreas del saber, en este caso la física, se puede refinar la iniciativa hasta convertirla en una idea materializable o por el contrario reconocer la imposibilidad de la ejecución de la misma.

Fase del proyecto final. En esta tercera fase del proceso de aula, los estudiantes consolidan la idea formulada con un modelo físico (a escala) y sustentan su iniciativa reconociendo algunos aspectos matemáticos y/o modelos que la describan. El estudiante presenta el modelo físico (Fig. 2) y relaciona matemáticamente los siguientes aspectos: 
Fig. 2. Modelo físico del diseo en la fase del proyecto final.

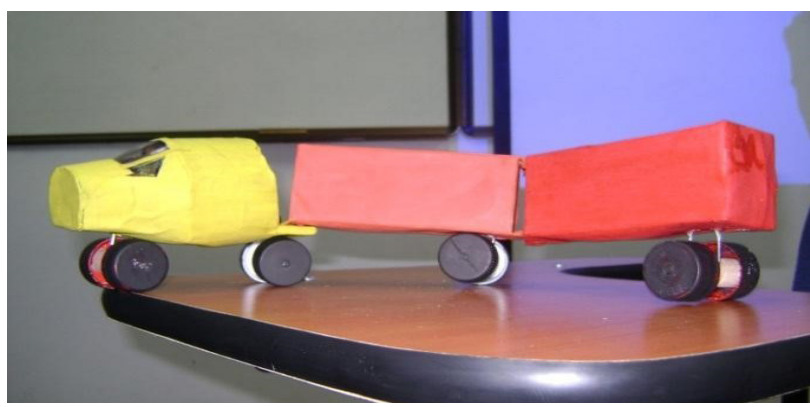

Fuente: Elaboración propia

- Líneas rectas que definen sus bordes, es decir, líneas como aristas. Su ecuación es; $\mathrm{y}=\mathrm{mx}+\mathrm{b}$ b; Elipses inclinadas en las vistas isométricas de los círculos de las caras de los cilindros. Su ecuación es $(\mathrm{x}-\mathrm{a})^{2}+\left(\mathrm{y}-\mathrm{b}^{2}=\mathrm{r}^{2} \mathrm{y}\right.$ un segmento de parábola en la cabina del camión, la cual tienen por ecuación $\mathrm{y}=\mathrm{ax}^{2}+\mathrm{bx}+\mathrm{c}$.

- Los tres ejes (X-Y-Z) que representados en el plano generan la impresión de Tridimensionalidad.

- Cilindros en sus llantas, rines, bisagras, luces. Prismas rectangulares en el conteiner. Rectángulos en sus caras y trapecios en sus ventanas laterales.

Estas descripciones llevan a pensar que los estudiantes no han logrado una "comprensión sobre los conceptos matemáticos"; puesto que solo enuncian algunos términos, de manera aislada y no se reconocen las relaciones entre las formas del modelo y su uso.

\section{Discusión}

Los trabajos desarrollados, parten de la elección de un problema propio del contexto, la formulación asociada al mismo y su posible solución a través del diseño y la matemática. Estos aspectos, se encuentran inscritos dentro de lo que se describe como Modelación Matemática propuesta por Blum et al.
(2007), Blum y Borromeo-Feri (2009), Villa-Ochoa \{Villa-Ochoa, 2007, La Modelación como Proceso en el Aula de Matemáticas: Un Marco de Referencia y un Ejemplo. \}(2007) entre otros. Sin embargo, pese a que existe un contexto, los estudiantes no se apropian de él y no establecen análisis de las condiciones. Es decir, el reconocimiento es solamente de aspectos "externos" pero no de variables que afectarían directamente la situación.

En este sentido, Barquero et al. (2007) establece que:

Lo importante para las instituciones universitarias es la "aplicación": lo primero es aprender a manejar los modelos matemáticos supuestamente únicos y luego ya se verá como "aplicarlos" a cada ámbito particular de trabajo. No se tiene en cuenta que, en muchas ocasiones, el modelo matemático que se pretende aplicar proviene de la matematización o modelización previa del sistema al cual queremos aplicar el modelo. En definitiva, se considera que la aplicación y la matematización (o modelización) son procesos independientes cuando, en realidad, son procesos inversos que se condicionan y dan sentido mutuamente (p. 2).

Por tanto, es necesario repensar los componentes micro-curriculares de tal forma que permita ampliar las conceptualizaciones matemáticas para que vayan más allá de unas formas y las ecuaciones "artificiales" para justificar de una manera superficial las matemáticas presentes en el diseño. De esta manera, se plantea la necesidad de buscar estrategias para que los estudiantes desarrollen ideas matemáticas que les permitan analizar la situación a la que se enfrentan y puedan, posteriormente, ser aplicadas como herramienta conceptual para resolver otros problemas (Trigueros, 2009). Esto conlleva a pensar en la Modelación Matemática, como una iniciativa donde el estudiante, a partir de sus intereses, pueda construir una "realidad", estructurarla, matematizarla y el profesor, se convierta en un agente problematizador de las producciones, de tal manera que genere reflexiones más profundas y refinamientos 
en los modelos producidos. Todo lo anterior como una respuesta a las exigencias actuales en la formación de ingenieros, que plantea la necesidad de un saber específico aplicado a un contexto.

\section{Referencias}

Barquero, B., Bosch, M. \& Gascón, J. (2007). La modelización matemática como instrumento de articulación de las matemáticas del primer ciclo universitario de Ciencias. Estudio de la dinámica de poblaciones. In L. Ruiz, Estepa, A. y García, F. (Ed.), Sociedad, escuela y matemáticas. Aportaciones de la Teoría Antropológica de lo Didáctico. (pp. 573-594).

Biembengut, M., \& Hein, N. (2004). Modelación matemática y los desafíos para enseñar matemática. Educación Matemática, 16(2), 105-125.

Blum, W., \& Borromeo-Ferri, R. (2009). Mathematical Modelling: Can It Be Taught And Learnt? Journal of Mathematical Modelling and Application, 1(1), 45-58.

Blum, W., Galbraith, P., Henn, H., \& Niss, M. (Eds). (2007). Modelling and Applications in Mathematics Education. The 14th ICMI Study (Vol. 10). New York: Springer.

Greer, B. (1997). Modelling reality in mathematics classrooms: The case of word problems. Learning and Instruction, 7(4), 293-307. doi: http://dx.doi.org/10.1016/ S0959-4752(97)00006-6

Kim, B. (2011). Putting context in context: an examination of the evidence for the enefits of 'contextualised' tasks autor. Revista Internacional de Ciencias y Educación Matemática, 9(2), 367-390.

Masingila, J., Davidenko, S., \& Prus-Wisniowska, E. (1996). Mathematics learning and practice in and out of school: A framework for connecting these experiences. Educational Studies in Mathematics, 31(1-2), 175-200. doi: 10.1007/ BF00143931

Trigueros, M. (2009). El uso de la Modelación en la Enseñanza de las Matemáticas. Innovación Educativa, 9(46), 75-87.

Villa-Ochoa, J. (2007). La Modelación como Proceso en el Aula de Matemáticas: Un Marco de Referencia y un Ejemplo. Tecno LóGicas, 19, 63-86.

Villa-Ochoa, J. A., \& Jaramillo, C. M. (2011). Sense of Reality through mathematical modeling. In G. Kaiser, W. Blum, R. Borromeo Ferri \& G. Stillman (Eds.), Trends in the teaching and learning of mathematical modelling ICTMA14, 701-711, New York: Springer. 